\title{
The effect of oxygen-plasma treatment on the mechanical andpiezoelectrical properties of ZnO nanorods
}

Mushtaque Hussain, Azam Khan, Omer Nur, Magnus Willander and Esteban Broitman

\section{Linköping University Post Print}

\section{Tweet}

N.B.: When citing this work, cite the original article.

Original Publication:

Mushtaque Hussain, Azam Khan, Omer Nur, Magnus Willander and Esteban Broitman, The effect of oxygen-plasma treatment on the mechanical andpiezoelectrical properties of $\mathrm{ZnO}$ nanorods, 2014, Chemical Physics Letters, (608), 235-238.

http://dx.doi.org/10.1016/j.cplett.2014.06.018

Copyright: Elsevier http://www.elsevier.com/

Postprint available at: Linköping University Electronic Press http://urn.kb.se/resolve?urn=urn:nbn:se:liu:diva-108547 


\title{
The effect of oxygen-plasma treatment on the mechanical and piezoelectrical properties of $\mathrm{ZnO}$ nanorods
}

Mushtaque Hussain*,1, Azam Khan', Omer Nur', Magnus Willander', Esteban Broitman'.

${ }^{1}$ Department of Science and Technology, Campus Norrköping, Linköping University, SE60174 Norrköping, Sweden

${ }^{2}$ Department of Physics, Chemistry and Biology (IFM), Linköping University, SE-58183 Linköping, Sweden

Correspondening author: mushtaque.hussain@liu.se

Phone number: +46-11-363652; Fax: +46-11-363270.

\begin{abstract}
:
We have studied the effect of oxygen plasma treatment on piezoelectric response and on the mechanical stability of $\mathrm{ZnO}$ nanorods synthesized on FTO by using ACG method. XRD and SEM techniques have shown highly dense and uniformly distributed nanorods. The piezoelectric properties and mechanical stability of as-grown and oxygen plasma treated samples were investigated by using nanoindentation technique. The comparison of loaddisplacement curves showed that the oxygen plasma treated samples are much stiffer and show higher generated piezo-voltage. This study demonstrates that the oxygen-plasma treatment is a good option to fabricate reliable and efficient nanodevices for enhanced generation of piezoelectricity.
\end{abstract}

Key words: $\mathrm{ZnO}$ nanorods, Oxygen plasma treatment, Nanoindentation, Piezoelectricity. 


\section{Introduction:}

The future direction of nanotechnology is based on the development of self-powered nanosystems due to their possible applications in real time and implantable biosensing, environmental monitoring, electromechanical systems and even personal electronics [1-3]. Basically, a self-powered nanosystem is an integration of nanodevices, functional components and a power source. The power source can be either a battery or harvested from the environment by converting mechanical, chemical, or thermal energy into electricity [4]. The main problems with batteries are lifetime, size, weight and toxicity of the materials they are built, while harvesting energy from environment is beneficial for an independent, wireless and sustainable operation of nanodevices [5]. In this context, a wide range of approaches for scavenging energy has been explored using thermoelectrics, mechanical vibration, and piezoelectric vibration $[6,7]$. Conversion of mechanical energy into electrical energy is more advantageous due to its abundance in our environment and unique fit for some applications. Therefore the use of a piezoelectric energy harvester, which is also known as nanogenerator (NG), is emerging as an effective strategy to develop self-powered nanosystems. Wang et al introduced the concept of $\mathrm{NG}$ by utilizing the piezoelectric properties of $\mathrm{ZnO}$ nanowires with an atomic force microscope (AFM) [8]. Different piezoelectric materials such as $\mathrm{ZnO}, \mathrm{GaN}$, $\mathrm{CdS}$, and $\mathrm{ZnS}$ are being investigated for the development of NGs, among which, $\mathrm{ZnO}$ proved to be the best due to some exceptional features. For example, it has the most diverse and abundant nano-structural configurations such as nanobelts, nanosprings, nanorings, nanohelices, etc [9-12].

The structural formation of $\mathrm{ZnO}$ can be described as a number of alternating planes composed of tetrahedrally coordinated $\mathrm{O}^{2-}$ and $\mathrm{Zn}^{2+}$ ions stacked along the $c$-axis. The oppositely charged ions produce positively charged $\mathrm{Zn}$ and negatively charged $\mathrm{O}$ polar surfaces that result in a normal dipole moment and spontaneous polarization along the $c$-axis. Hence by utilizing semiconducting-piezoelectric coupled properties of $\mathrm{ZnO}$, scientists were able to develop many self-powered devices including NGs [8, 13], piezoelectric field effect transistors [14], piezoelectric diodes [15] and piezoelectric chemical sensors [16]. In ZnO, the two most common defects are oxygen and zinc vacancies. The oxygen vacancy has lower formation energy than the zinc interstitial and dominates in zinc rich growth conditions. Therefore by controlling/reducing the quantity of oxygen vacancies, it is possible to modify the piezoelectric properties of $\mathrm{ZnO}$ [17]. Hydrothermal growth of $\mathrm{ZnO}$ is one of the most powerful low-cost, low-temperature and extensively used technique both at large-scale and nano-scale [18-20]. Among different hydrothermal techniques, aqueous chemical growth 
(ACG) method is more suitable because the growth temperature is around $80-100^{\circ} \mathrm{C}$, so the substrate can be any kind of material such as graphene, common paper, flexible plastic, textile fabric, glass etc. One of the unique advantages is that the nanowires and nanorods grow along the $c$-axis (i.e., in the polar direction) and are uniaxially aligned.

Several techniques have been utilized by the researchers to record the piezoelectric response from $\mathrm{ZnO}$ nanorods including interferometry [21], scanning tunneling microscopy [22], scanning probe microscopy (SPM) [23], atomic force microscopy (AFM) [24] and piezoresponse force microscopy (PFM) [25]. Recently, we have introduced a nanoindentation technique as a new and versatile method for studying piezoelectric properties of $\mathrm{ZnO}$ nanorods [26]. We have also used the same technique for the current study.

\section{Experimental:}

In order to avoid any kind of contamination, we performed all the experimental work in clean room environment. Fluorine doped tin oxide (FTO) coated glass substrates (Sigma-Aldrich) were cleaned in ultrasonic bath with acetone, isopropanol and washed with deionized water and dried by flow of nitrogen gas. For the growth of well aligned $\mathrm{ZnO}$ nanorods, zinc acetate dihydrate $\left[\mathrm{Zn}\left(\mathrm{CH}_{3} \mathrm{COO}\right)_{2} \cdot 2 \mathrm{H}_{2} \mathrm{O}\right]$ (Sigma-Aldrich, 99.9\% purity) used as a seed solution was spin-coated 2-3 times (30 seconds each time) at 3000 r.p.m on the FTO coated glass substrate and consequently annealed at $100^{\circ} \mathrm{C}$ for $10-15$ minutes for good adhesion of seed particles. In a beaker an equimolar (0.075) solution of zinc nitrate hexahydrate $\left[\mathrm{Zn}\left(\mathrm{NO}_{3}\right)_{2} \cdot 6 \mathrm{H}_{2} \mathrm{O}\right]($ Sigma Aldrich, 98\% purity) and hexamethylenetetramine $\left[\mathrm{C}_{6} \mathrm{H}_{12} \mathrm{~N}_{4}\right]$ (Sigma Aldrich, 99\% purity) was prepared in $75 \mathrm{ml}$ deionized water. The substrates decorated with $\mathrm{ZnO}$ particles were placed in the beaker with the help of a Teflon sample holder. The beaker was kept in the preheated electric oven at $95^{\circ} \mathrm{C}$ for 5-6 hours. After the growth, the substrates having $\mathrm{ZnO}$ nanostructures were carefully washed with the deionized water in order to remove the unwanted residual particles from the surface of nanostructures and then dried in air at room temperature.

In order to reduce the defects level in the grown $\mathrm{ZnO}$ nanorods, some samples were treated with the oxygen plasma to observe its effect on the piezoelectric property of $\mathrm{ZnO}$ nanorods.

The $\mathrm{O}^{2-}$ plasma treatment was performed by using a reactive ion etching (RIE) system (SAMCO, RIE-10RU) equiped with parallel plate type plasma reactor chamber. The oxygen plasma was induced during three minutes using an applied RF power of $250 \mathrm{~W}$, RF frequency of $13.56 \mathrm{MHz}$, a gas flow of $400 \mathrm{sccm}$ and a pressure of $600 \mathrm{~Pa}$. 
The morphology of the grown $\mathrm{ZnO}$ nanorods was investigated by the help of field emission scanning electron microscopy (FESEM) model LEO 1550 Gemini microscope running at 15 $\mathrm{kV}$. X-ray diffraction (XRD) technique was used for the investigation of crystal quality of $\mathrm{ZnO}$ nanorods by using a Phillips PW 1729 powder diffractometer equipped with $\mathrm{Cu} \mathrm{K} \alpha$ radiation $(\lambda=1.5418 \AA$ ) having a generator voltage of $40 \mathrm{kV}$ and a current of $40 \mathrm{~mA}$. To analyse the electromechanical properties of the as grown $\mathrm{ZnO}$ nanorods, nanoindentation was performed by using a Triboindenter TI-950 (Hysitron) with a conductive boron-doped diamond Berkovich tip of $\sim 3 \Omega \mathrm{cm}$ resistivity and $\sim 200 \mathrm{~nm}$ diameter.

\section{Results and Discussion:}

In figure 1 typical SEM images of as-grown and oxygen plasma treated $\mathrm{ZnO}$ nanorods on FTO glass substrate are shown. It is clear in figure 1 (a) that as grown $\mathrm{ZnO}$ nanorods are highly dense, that is around $\sim 13000^{-} 15000$ nanorods per square millimetre. The nanorods are well aligned, uniformly distributed over the surface and perpendicularly oriented on the FTO glass substrate. The size of the nanorods is rather uniform with a length of $\sim 1 \mu \mathrm{m}$ and a diameter of $\sim 200 \mathrm{~nm}$ as shown in the inset of figure 1 (a). The figure 1(b) is showing the SEM image after receiving oxygen plasma treatment. It can be inferred that oxygen plasma treatment did not affect the density, alignment and uniformity of the nanorods.

The crystalline study of as-grown and oxygen plasma treated $\mathrm{ZnO}$ nanorods by XRD (Figure 2) shows that all the diffraction pattern peaks could be assigned to crystalline $\mathrm{ZnO}$ as per JCPDS card no. (36-1451). The only difference in both spectra lies in the intensity of (002) peak, which is probably due to the plasma treatment. This peak at $34.4^{\circ}$ demonstrates the caxis orientation of the nanorods, while other present peaks are just the reflections for $\mathrm{ZnO}$. This study indicates the formation of pure phase of $\mathrm{ZnO}$ nanostructures and no other crystallized phase was observed.

Piezoelectric tests were performed by nanoindentation testing using the configuration shown in Fig. 3. The piezoelectric effect is measured by applying a DC voltage in the range 0 to -40 $\mathrm{V}$ while there is a low applied force to the sample of $50 \mu \mathrm{N}$, enough to allow the tip to be always in physical contact with the $\mathrm{ZnO}$ nanorods. This approach is similar to the method previously suggested by Rar et al. for polycrystalline lead zirconate titanate (PZT) and $\mathrm{BaTiO}_{3}$ piezoceramics [27] but in our case the applied voltage is DC instead of AC.

Figure 4 is revealing the generated piezo-voltage against different applied forces. Under indentations with loads between 15 and $450 \mu \mathrm{N}$, oxygen plasma treated sample has generated higher voltage than as-grown one. In particular the oxygen plasma treatment seems specially 
to increase the generated voltage between 30 and $200 \mu \mathrm{N}$. The improvement in the piezoelectric potential after oxygen plasma treatment can be attributed to the reduction in the defects level of the $\mathrm{ZnO}$ nanorods. The generated oxygen ions and radicals from the plasma diffused into the $\mathrm{ZnO}$ nanorods and occupied the oxygen vacancies, resulting in a decrease of the carrier concentration. The oxygen plasma treatment also decreased the free carrier concentration in the $\mathrm{ZnO}$ nanorods, resulting in an increase of the piezoelectric potential. Moreover, the induction of oxygen plasma contributed to the filling of vacancies as well, which improved the piezoelectric properties of $\mathrm{ZnO}$ nanorods by rendering more polarization of charges throughout the applied force. Furthermore, the decrease of defects can contribute to the improved elastic property/better deflection of $\mathrm{ZnO}$ nanorods. These results are supported by our recent publication, where we have shown that the increase in piezoelectric response is due to the filling of few oxygen vacancies after the induction of oxygen plasma that also improved the crystallinity of the $\mathrm{ZnO}$ nanorods [17]. In these experiments $\mathrm{ZnO}$ nanorods have been synthesized on single layer graphene substrate and the piezo-response was measured by using an atomic force microscope (AFM) technique. While in the AFM piezocharacterization, the NRs are laterally deformed to generate the voltage, in the present experiments the NRs are controlled-load pressed from the top, obtaining more precise information about the effect of the plasma treatment.

Figure 5 is showing the load-displacement curves for the nanoindentation of bare FTO glass substrate and $\mathrm{ZnO}$ nanorods with and without oxygen plasma treatment. All samples were loaded to a prescribed force and then unloaded in a force controlled mode at room temperature. Under a maximum applied load of $400 \mu \mathrm{N}$, the bare FTO substrate showed a tip penetration of $\sim 75 \mathrm{~nm}$ (figure 5(a)). The presence of a soft surface coating $\sim 30-40 \mathrm{~nm}$ thick can be related to the conductive layer on the glass. For the $\mathrm{ZnO}$ NRs coated substrates the penetration of the tip was about $\sim 650 \mathrm{~nm}$. The comparison of load-displacement curves for both NRs samples show that the oxygen plasma treated samples are stiffer than the as grown ones, because the tip needs to apply more force after $300 \mathrm{~nm}$ penetrations to buckle/bend [2830] the plasma-treated nanorods.

\section{Conclusion:}

In this paper we demonstrated the experimental observation of the stability and piezoelectric generation of well aligned $\mathrm{ZnO}$ nanorods grown by using the low temperature aqueous chemical growth method on FTO coated glass substrate. The comparative results of ZnO 
nanorods with and without oxygen plasma treatment showed that, after the oxygen plasma treatment, the $\mathrm{ZnO}$ nanorods become stiffer and also generated higher piezo-voltage. More work still is needed in terms of adjusting plasma experimental parameters to understand the induced oxygen in order to maximize the piezo-generated response.

\section{Acknowledgement:}

Esteban Broitman acknowledges the Swedish Government Strategic Research Area in Materials Science on Functional Materials at Linköping University (Faculty Grant SFO-MatLiU \# 2009-00971).

\section{References:}

[1]. F. Patolsky, B. P. Timko, G. Zheng, C. M. Lieber, MRS Bull. 2007, 32, 142.

[2]. F. Patolsky, B. P. Timko, G. Yu, Y. Fang, A. B. Greytak, G. Zheng, C. M. Lieber, Science 2006, 313, 1100.

[3]. P. Pauzauskie, P. Yang, Mater. Today 2006, 9, 36.

[4]. J. A. Paradiso, T. Starner, Pervasive Computing 2005, 5, 18.

[5]. N. S. Shenck, J. A. Paradiso, IEEE MICRO 2001, 21, 30.

[6]. J. A. Paradiso, T. Starner, IEEE Pervasive Comput. 2005, 4, 18.

[7]. E. S. Leland, J. Baker, E. Carleton, E. Reilly, E. Lai, B. Otis, J. M. Rabaey, P. K. Wright, V. Sundararajan, IEEE Pervasive Comput. 2005, 14, 28.

[8]. Z. L. Wang, J. H. Song, Science 2006, 312, 242.

[9]. Z. W. Pan, Z. R. Dai, Z. L. Wang, Science 2001, 209, 1947.

[10]. X. Y. Kong, Z. L. Wang, Nano Lett. 2003, 3, 1625.

[11]. X. Y. Kong, Y. Ding, R. S. Yang, Z. L.Wang, Science 2004, 303, 1348.

[12]. P. X. Gao, Y. Ding, W. J. Mai, W. L. Hughes, C. S. Lao, Z. L. Wang, Science 2005, 309, 1700.

[13]. X. D. Wang, J. H. Song, J. Liu, Z. L. Wang, Science 2007, 316, 102.

[14]. X. D. Wang, J. Zhou, J. H. Song, J. Liu, N. S. Xu, Z. L. Wang, Nano Lett. 2006, 6, 2768.

[15]. J. H. He, C. L. Hsin, J. Liu, L. J. Chen, Z. L. Wang, Adv. Mater. 2007, 19, 781.

[16]. C. S. Lao, Q. Kuang, Z. L. Wang, C. M. Park, Y. Deng, Appl.Phys. Lett. 2007, 90, 262107. 
[17]. M. Hussain, M. A. Abbasi, Z. H. Ibupoto, O. Nur, M. Willander, Phys. Status Solidi A 2014, 211(2), 455.

[18]. S. Xu , Z.L. Wang, Nano Res. 2011, 4, 1013.

[19]. Y. G. Wei , W. Z. Wu , R. Guo, D. J. Yuan, S. Das , Z. L. Wang, Nano Lett. 2010, 10, 3414.

[20]. S. Xu , C. S. Lao, B. Weintraub , Z. L. Wang, J. Mater. Res. 2008, 32, 2072.

[21]. A. L. Kholkin, C. Wutchrich, D. V. Taylor, N. Setter, Rev. Sci. Instrum. 1996, 67, 1935.

[22]. R. Winters, M. Reinermann, C. Enss, G. Weiss, S. Hunklinger, Conf. Proc. 1995, 13, 1316.

[23]. A. G. Agronin, Y. Rosenwaks, G. I. Rosenman, Nano Lett. 2003, 3, 169.

[24]. A. Khan, M. A. Abbasi, M. Hussain, Z. H. Ibupoto, J. Wissting, O. Nur, M. Willander, Appl. Phys. Lett. 2012, 101, 193506.

[25]. V. V. Shvartsman, A. L. Kholkin, N. A. Pertsev, Appl. Phys. Lett. 2002, 81, 3025.

[26]. E. Broitman, M. Y. Soomro, J. Lu, M. Willander, L. Hultman, Phys.Chem. Chem. Phys. 2013, 15, 11113.

[27]. A. Rar, G. Pharr, W. Oliver, E. Karapetian, S. Kalinin, J. Mater. Res. 2006, 21, 552.

[28]. M. Riaz, A. Fulati, Q. X. Zhao, O. Nur, M. Willander, P. Klason, Nanotechnology. 2008, $19,415708$.

[29]. M. Riaz, A. Fulati, L. L. Yang, O. Nur, M. Willander, P. Klason, J. App.Phys. 2008, $104,104306$.

[30]. M. Riaz, A. Fulati, G. Amin, N. H. Alvi, O. Nur, M. Willander, J. App.Phys. 2009, 106 , 034309. 


\section{Figure Captions:}

Figure 1. SEM images of $\mathrm{ZnO}$ nanorods grown on FTO glass substrate.

(a) without oxygen plasma treatment. (b) with oxygen plasma treatment.

Figure 2. XRD spectra of $\mathrm{ZnO}$ nanorods grown on FTO glass substrate

(a) without oxygen plasma treatment. (b) with oxygen plasma treatment.

Figure 3. Schematic drawing of the nanoindentation experimental setup.

Figure 4. Generated piezo-voltage without and with oxygen plasma treatment as a function of maximum applied load. The dotted lines are just for guiding the eyes .

Figure 5. Load-displacement curve recorded by using nanoindentation technique. The used probe was a boron-doped diamond Berkovich tip and the measurements were performed in the load-control mode. (a) Bare FTO glass substrate. (b) NR without oxygen plasma treatment. (c) NR with oxygen plasma treatment. 
Figure 1.

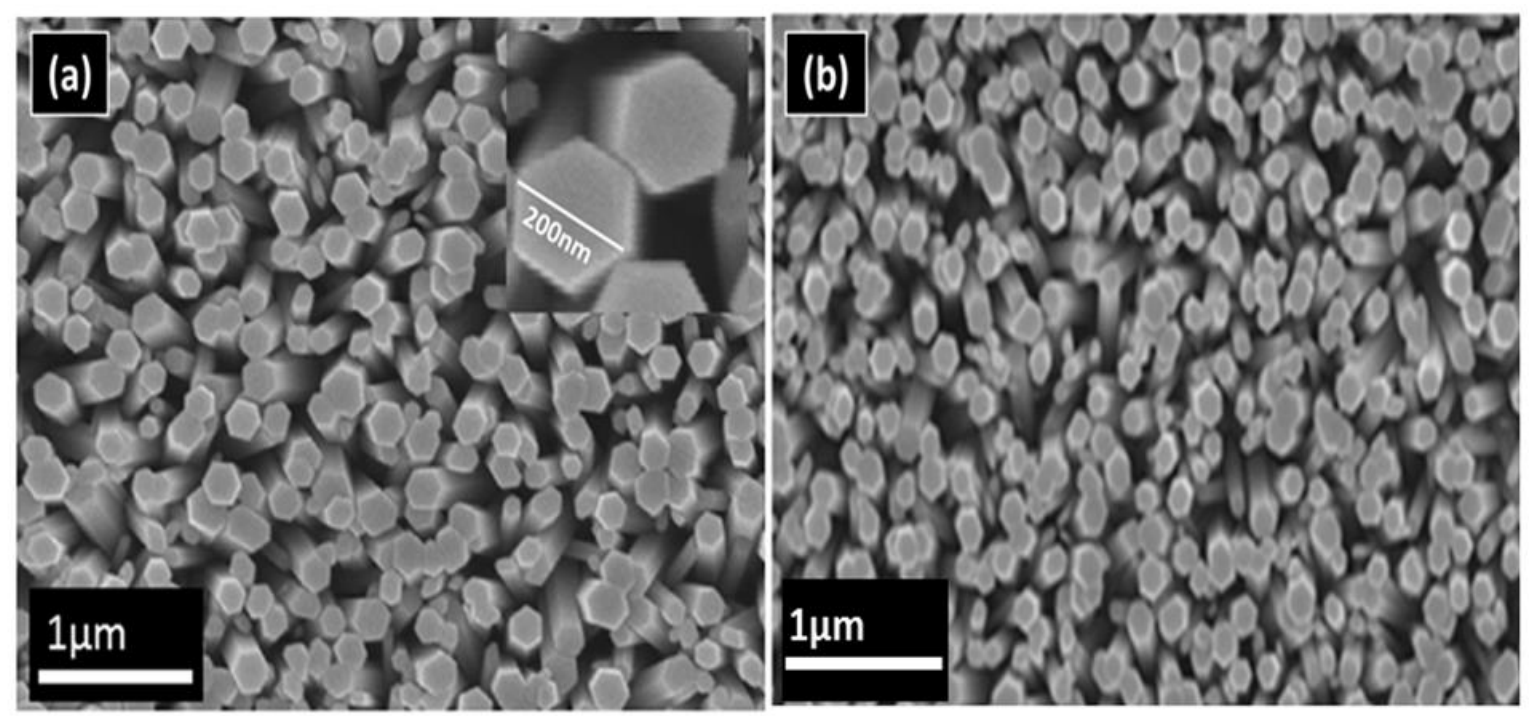

Figure 2.
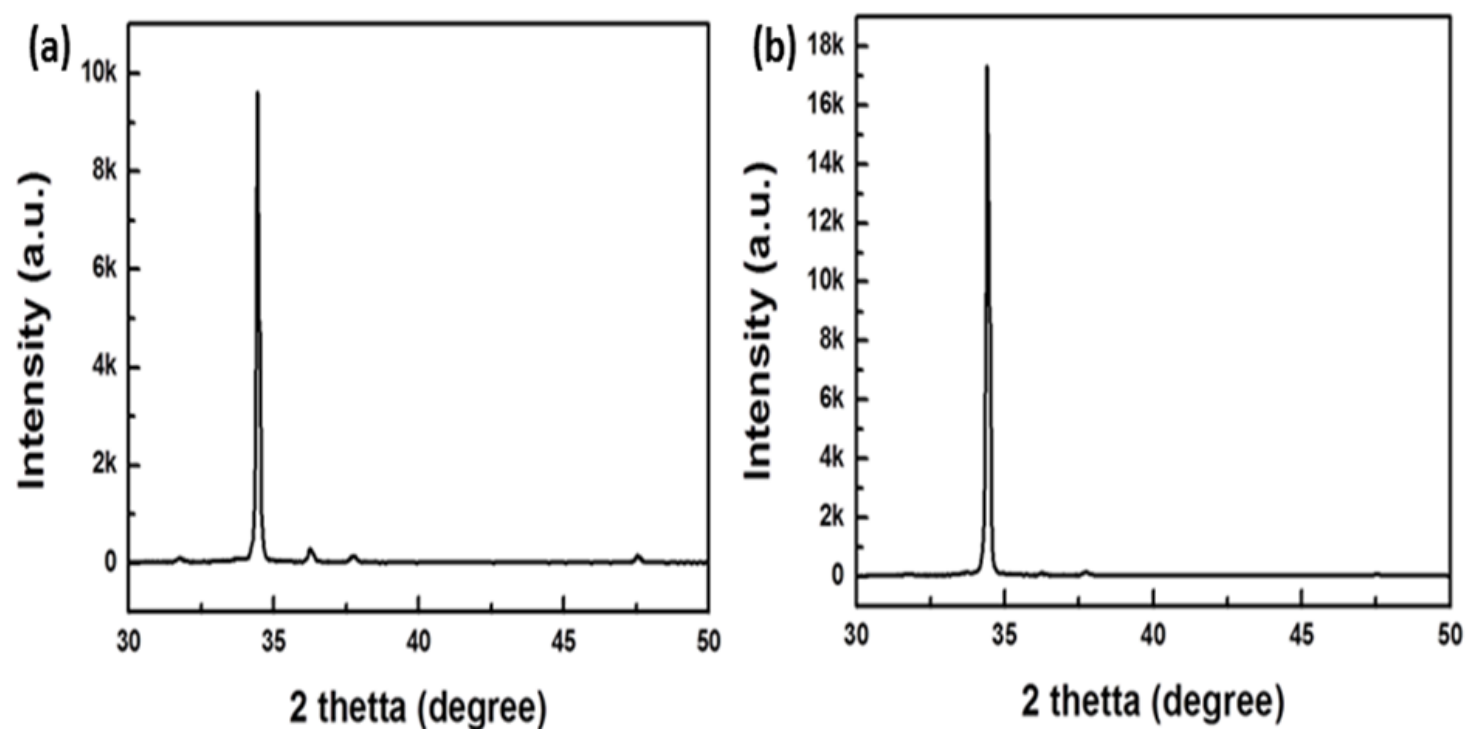
Figure 3.

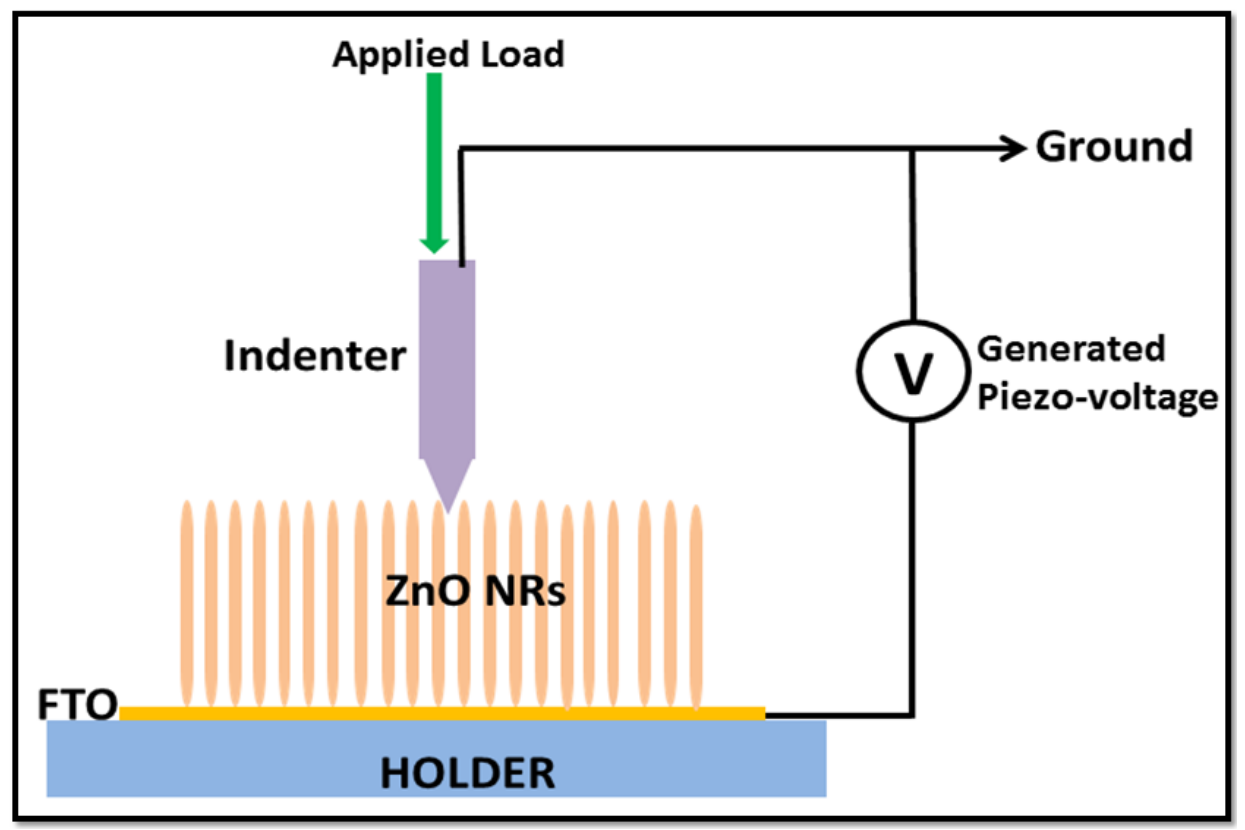

Figure 4.

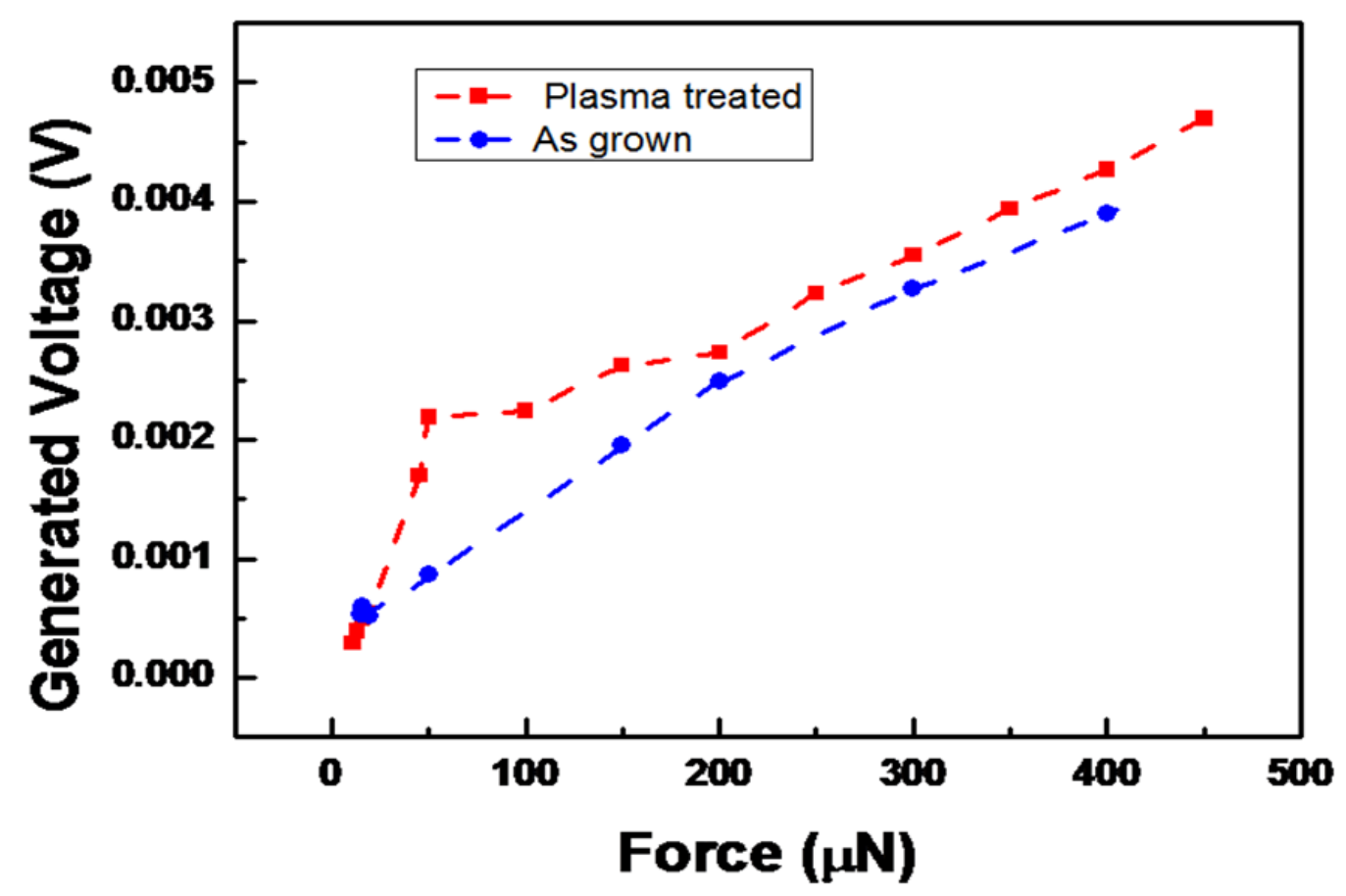


Figure 5.
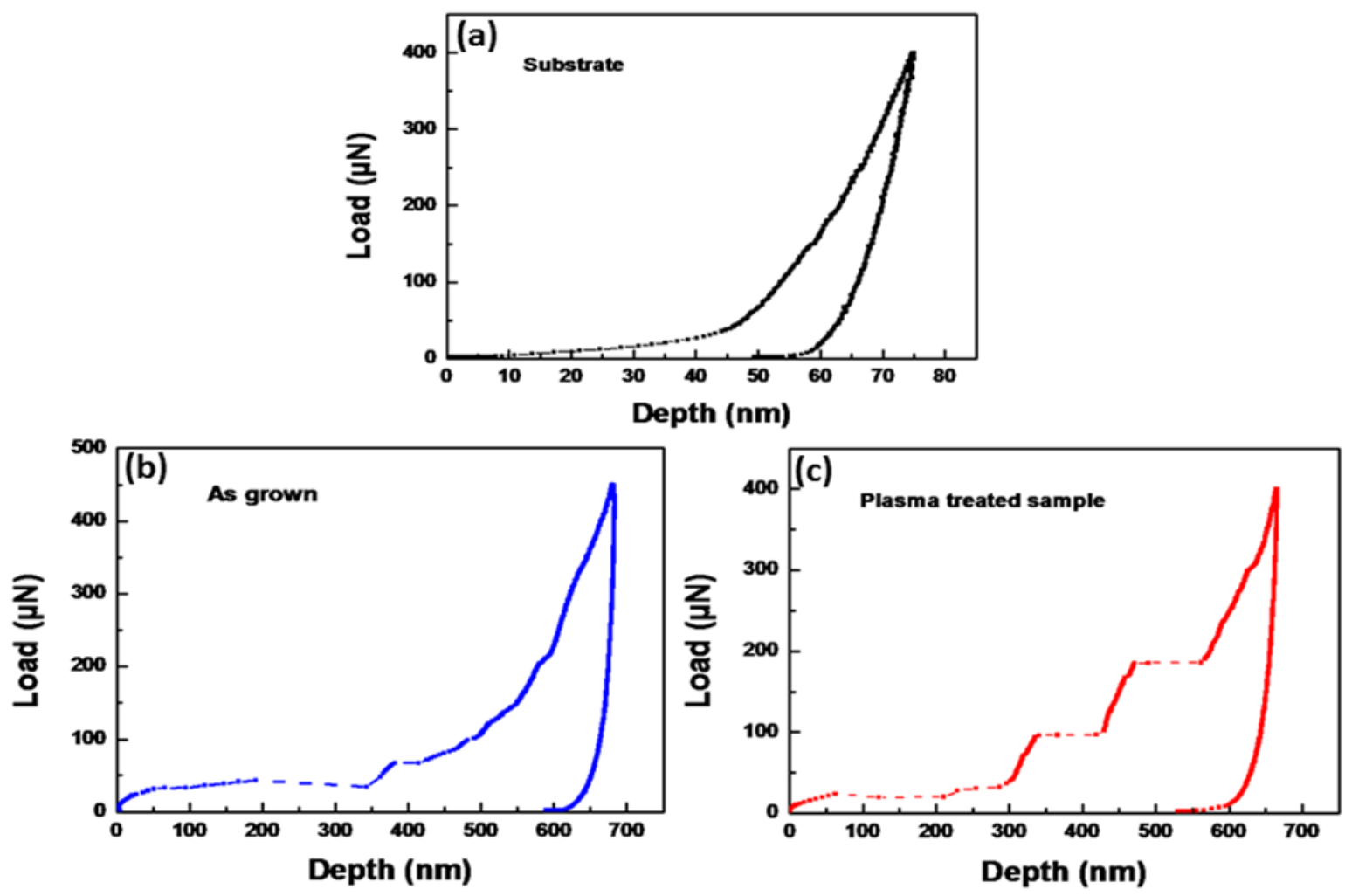